\title{
Détermination expérimentale de l'efficacité de blindage de matériaux par différentes méthodes
}

\author{
Adrien Meynard, Françoise Paladian, Sébastien Girard \\ adrien.meynard@ens-cachan.fr \\ Université Clermont Auvergne, Institut Pascal, BP 10448, F-63000 CLERMONT-FERRAND \\ CNRS, UMR 6602, Institut Pascal, F-63171 AUBIÈRE
}

\begin{abstract}
Résumé
La démarche proposée dans cet article peut constituer le cadre de travaux pratiques de Master 2. L'objectif est d'initier les étudiants à la pratique d'expérimentations en haute fréquence pour des applications en compatibilité électromagnétique. Deux moyens expérimentaux sont utilisés pour la caractérisation électromagnétique de matériaux sur une large gamme de fréquences : les cellules anéchoïques et les chambres réverbérantes à brassage de modes. L'objectif de ces deux TP sera la mesure d'efficacité de blindage d'échantillons de matériaux plans.

Dans un premier temps, nous décrivons l'approche théorique qui permet d'introduire la notion d'efficacité de blindage. Dans un second temps, la cellule anéchoïque nous permettant de mesurer l'efficacité de blindage d'un matériau soumis à une onde plane d'incidence normale est mise en œuvre. Nous présentons également la chambre réverbérante à brassage de modes qui permet de soumettre l'échantillon à une infinité d'ondes planes d'incidences quelconques. Enfin, nous comparons les résultats obtenus par les deux méthodes, l'analyse des grandeurs mesurées permettant de conclure sur les avantages et inconvénients de chacune des techniques.
\end{abstract}

Mots-clés : électromagnétisme, compatibilité électromagnétique, efficacité de blindage, mesures, antennes, analyseur de réseau, travaux pratiques d'hyperfréquences.

\section{INTRODUCTION}

La compatibilité électromagnétique traite des effets de couplage électromagnétique entre différents circuits électroniques, ou électriques. Ces effets proviennent de la propagation de signaux électriques par des matériaux conducteurs et, du rayonnement d'ondes électromagnétiques à travers des milieux de transmission (air, diélectriques...). C'est pourquoi, il apparaît nécessaire de protéger les équipements électroniques ou d'autres éléments sensibles contre les champs rayonnés extérieurs. Pour cela, les zones que l'on souhaite protéger sont isolées à l'intérieur d'enceintes. Celles-ci constituent des blindages électromagnétiques, elles sont réalisées à l'aide d'un matériau (souvent métallique) permettant d'atténuer l'amplitude des champs électromagnétiques au sein de l'enceinte. L'efficacité de blindage est une notion qui permet de quantifier la capacité d'une enceinte ou d'un dispositif à isoler des rayonnements électromagnétiques extérieurs.

Par la suite, nous nous intéressons à la détermination de l'efficacité de blindage d'échantillons de matériaux plans. Ces matériaux sont, par exemple, des isolants métalliques que l'on place sur les parois de pièces que l'on souhaite isoler des champs rayonnés extérieurs. Dans la première partie, nous décrivons l'approche théorique re- lative à la caractérisation de l'efficacité de blindage de matériaux plans. Nous présentons ensuite deux méthodes de mesures d'efficacité de blindage sur ce type d'échantillons. Dans la deuxième partie,la première méthode utilisée qui consiste à générer une onde plane dans une cellule anéchoïque est détaillée. Dans la troisième partie, nous caractérisons la seconde méthode qui met en œuvre une chambre réverbérante à brassage de modes. Enfin, nous comparons les deux méthodes utilisées en vue d'en extraire les avantages et les inconvénients.

Vis-à-vis des objectifs pédagogiques de cette étude, la partie théorique doit être présentée préalablement aux TP. Par la suite, les mesures de gain à vide sont effectuées et sont communes aux deux TP. Enfin, les mesures d'efficacités de blindages selon les deux méthodes peuvent être exposées dans deux TP différents.

\section{L'EFFICACITÉ DE BLINDAGE}

L'efficacité de blindage permet de quantifier l'atténuation du champ électromagnétique produite par un objet donné. Pour cela, considérons, comme illustré figure 1, une onde incidente qui illumine l'objet que l'on souhaite caractériser. Cette onde peut alors subir une réflexion ou 
pénétrer dans le blindage. Une partie de cette onde sera alors atténuée en raison de l'absorption dans le matériau, ce phénomène devant être associé aux réflexions multiples (allers-retours de l'onde). Finalement, une partie de l'onde incidente traverse cet objet : il s'agit de l'onde transmise.

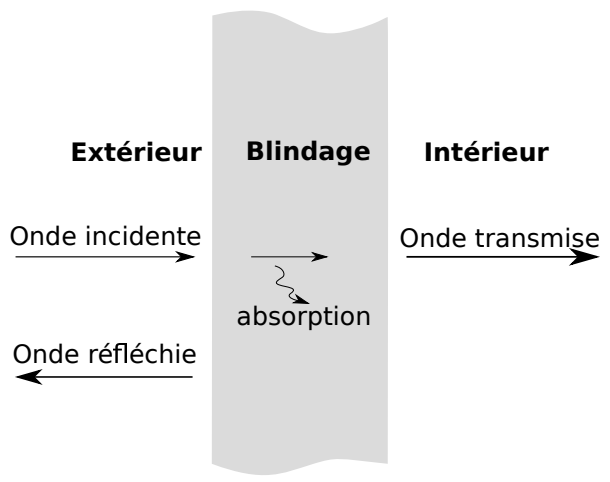

FIGURE 1 - Efficacité de blindage.

On définit alors l'onde externe qui est la superposition des ondes incidente et réfléchie présentes à l'extérieur du blindage. Celle-ci peut être décrite localement par les champs électrique $\mathbf{E}_{\mathbf{e x t}}$ et magnétique $\mathbf{H}_{\mathbf{e x t}}$ et, globalement par la puissance externe $\mathscr{P}_{\text {ext }}$ au travers d'une surface donnée. De même, pour l'onde interne, présente à l'intérieur du blindage, on définit $\mathbf{E}_{\text {int }}, \mathbf{H}_{\text {int }}$ et, $\mathscr{P}_{\text {int }}$.

Ayant introduit ces phénomènes, nous pouvons maintenant donner la définition de trois efficacités de blindage (exprimées en décibels) relatives aux différentes grandeurs décrivant l'onde électromagnétique [2] :

- l'efficacité de blindage en champ électrique :

$$
E B_{E}=20 \log \left(\frac{\left\|\mathbf{E}_{\mathbf{e x t}}\right\|}{\left\|\mathbf{E}_{\mathbf{i n t}}\right\|}\right) ;
$$

- l'efficacité de blindage en champ magnétique :

$$
E B_{H}=20 \log \left(\frac{\left\|\mathbf{H}_{\text {ext }}\right\|}{\left\|\mathbf{H}_{\text {int }}\right\|}\right) ;
$$

- l'efficacité de blindage en puissance (la puissance s'exprime en fonction des deux grandeurs précédentes par $\mathscr{P}=\iint_{\Sigma}(\mathbf{E} \times \mathbf{H}) \cdot \mathbf{n d} \Sigma$, où $\Sigma$ est la surface de mesure et $\mathbf{n}$ le vecteur unitaire normal à cette surface) :

$$
E B_{P}=10 \log \left(\frac{\mathscr{P}_{\text {ext }}}{\mathscr{P}_{\text {int }}}\right) .
$$

Les dispositifs de mesure que l'on va mettre en œuvre ne vont pas permettre de connaître l'efficacité de blindage d'un matériau en un point donné de la surface. En effet, nous ne pouvons mesurer que la puissance au travers d'une surface donnée. Ainsi, les mesures ne permettent d'obtenir que l'efficacité de blindage en puissance.
Il peut être intéressant de considérer une onde plane. En effet, les champs $\mathbf{E}$ et $\mathbf{H}$ étant uniformes dans un plan perpendiculaire à la direction de propagation, les efficacités de blindages locale et globale sont égales.

En effet, l'onde se propageant dans le vide a pour propriété d'être TEM (Transverse ElectroMagnétique). Ceci nous permet d'écrire $\mathbf{E} \times \mathbf{H}=\|\mathbf{E}\| .\|\mathbf{H}\| \mathbf{n}$. Il suffit ensuite de placer l'échantillon à tester au niveau du plan d'onde. En effet :

$$
\begin{aligned}
\mathscr{P} & =\iint_{\Sigma}(\mathbf{E} \times \mathbf{H}) \cdot \mathbf{n} \mathrm{d} \Sigma \\
& =(\mathbf{E} \times \mathbf{H}) \cdot \mathbf{n} \Sigma \\
& =\|\mathbf{E}\| \cdot\|\mathbf{H}\| \cdot \Sigma .
\end{aligned}
$$

On a alors :

$$
\begin{aligned}
E B_{P} & =10 \log \left(\frac{\mathscr{P}_{\text {ext }}}{\mathscr{P}_{\text {int }}}\right) \\
& =10 \log \left(\frac{\left\|\mathbf{E}_{\text {ext }}\right\| \cdot\left\|\mathbf{H}_{\text {ext }}\right\| \cdot \Sigma}{\left\|\mathbf{E}_{\text {int }}\right\| \cdot\left\|\mathbf{H}_{\text {int }}\right\| \cdot \Sigma}\right) \\
& =\frac{1}{2}\left(20 \log \left(\frac{\left\|\mathbf{E}_{\text {ext }}\right\|}{\left\|\mathbf{E}_{\text {int }}\right\|}\right)+20 \log \left(\frac{\left\|\mathbf{H}_{\text {ext }}\right\|}{\left\|\mathbf{H}_{\text {tr }}\right\|}\right)\right) \\
& =\frac{1}{2}\left(E B_{E}+E B_{H}\right) .
\end{aligned}
$$

En supposant identiques les milieux de propagation de l'onde TEM externe et interne au blindage (par exemple, de l'air), on a la relation :

$$
\|\mathbf{E}\|=\eta\|\mathbf{H}\|,
$$

avec $\eta$ l'impédance caractéristique du milieu de propagation. Dans ce cas :

$$
\begin{aligned}
E B_{E} & =20 \log \left(\frac{\left\|\mathbf{E}_{\text {ext }}\right\|}{\left\|\mathbf{E}_{\text {int }}\right\|}\right) \\
& =20 \log \left(\frac{\eta\left\|\mathbf{H}_{\text {ext }}\right\|}{\eta\left\|\mathbf{H}_{\text {int }}\right\|}\right) \\
& =E B_{H} .
\end{aligned}
$$

Finalement, on a :

$$
E B_{P}=E B_{E}=E B_{H} .
$$

\section{LA CELLULE ANÉCHOÏQUE}

Le cellule utilisée pour déterminer l'efficacité de blindage des matériaux testés doit permettre de générer une onde électromagnétique plane sur une gamme de fréquence la plus importante possible. 


\subsection{Principe}

Le schéma de principe de la cellule est donné figure 2.

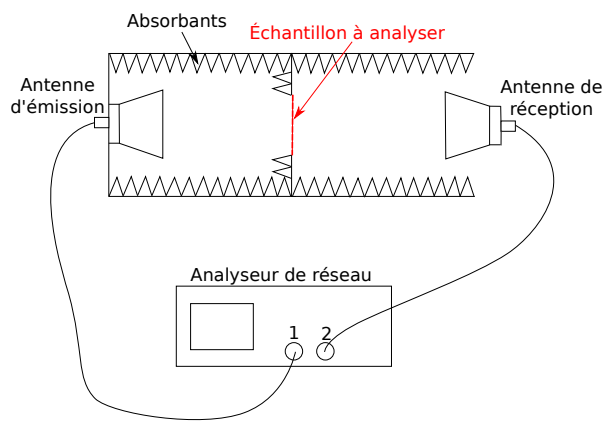

FIGURE 2 - Schéma de principe du dispositif.

Ce dispositif permet donc de mesurer l'efficacité de blindage d'échantillons de matériaux présentés sous forme plane. Il peut, par exemple, être utilisé pour caractériser un textile. De plus, nous pouvons utiliser plusieurs panneaux, de dimensions différentes, munis d'une ouverture (figure 3). Il est par conséquent possible de caractériser des échantillons de taille diverses.

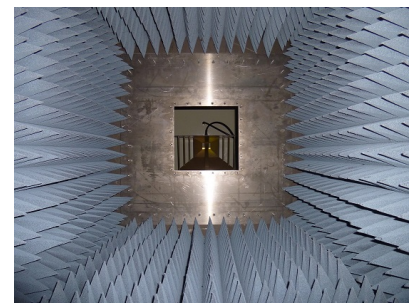

FiguRE 3 - Antenne d'émission vue depuis l'antenne de réception.

Dans la première partie de la chambre semianéchoïque, une antenne cornet est disposée. Cette antenne constitue l'antenne émettrice qui génère une onde électromagnétique qui va illuminer le matériau dont un souhaite déterminer l'efficacité de blindage. Le rôle des absorbants placés sur les parois de la chambre est de supprimer toute réflexion de l'onde afin de conserver la structure d'onde plane au niveau du matériau. Ces absorbants sont efficaces à partir d'une fréquence liée aux dimensions et aux matériaux des éléments constituants.

Les premiers éléments importants de ce système à caractériser sont les deux antennes (émission et réception). En effet, celles-ci doivent garantir des caractéristiques permettant les mesures envisagées dans une large bande de fréquence.

\subsubsection{Les antennes}

Les antennes utilisées sont des antennes cornets SH 400 de la marque Satimo (figure 4).

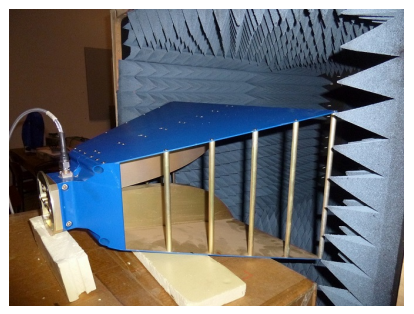

FIGURE 4 - Antenne de réception.

D'après les données techniques tracées figure 5 , ces antennes ont une bande passante suffisamment large pour émettre ou recevoir une onde électromagnétique de puissance suffisante dans une gamme de fréquence allant de $400 \mathrm{MHz}$ à $6 \mathrm{GHz}$. En effet, le coefficient de réflexion de l'antenne, reste inférieur à $-10 \mathrm{~dB}$ dans cette bande de fréquence, ce qui signifie qu'une majorité de la puissance reçue par l'antenne d'émission est rayonnée vers le milieu de propagation. Par réciprocité, dans cette gamme de fréquence, une majorité de la puissance incidente à l'antenne de réception est transmise vers le port 2 de l'analyseur de réseau.

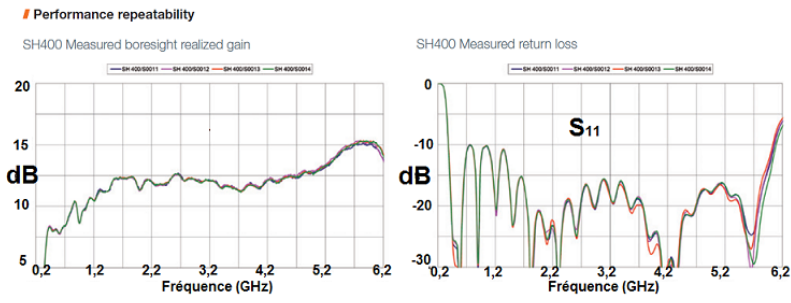

FIGURE 5 - Caractéristiques des antennes utilisées [6].

\subsubsection{L'analyseur de réseau}

L'analyseur de réseau utilisé possède une certaine dynamique de mesure. Celle ci-correspond au rapport entre la puissance maximale injectée au port 1 et la puissance minimale que mesurée au port 2.

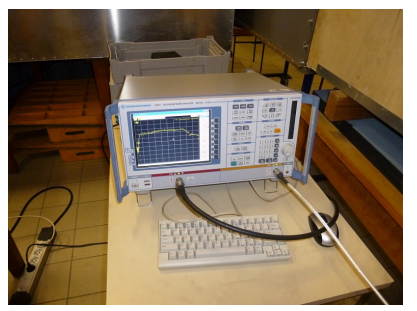

FIgURE 6 - Analyseur de réseau. 
On se place dans les conditions de mesure suivantes :

- la puissance maximale $\mathscr{P}_{M}$ que l'on peut émettre est de $20 \mathrm{dBm}$;

- la puissance minimale $\mathscr{P}_{m}$ mesurable par le port 2 dépend de la bande passante du filtre d'analyse : on choisit une largeur de $100 \mathrm{~Hz}$, d'où la possibilité de mesurer une puissance de $-100 \mathrm{dBm}$ (soit $\left.10^{-13} \mathrm{~W}\right)$;

Dans ces conditions, le gain minimum mesurable hors bruit intrinsèque à l'appareil est de $\mathscr{P}_{M}-\mathscr{P}_{m}=-120 \mathrm{~dB}$

Ces conditions de mesure étant définies, il s'agit maintenant de déterminer quelles sont les efficacités de blindages maximales mesurables au moyen de ce dispositif.

\subsection{Fonctionnement à vide}

Nous souhaitons vérifier, dans un premier temps, que l'onde au niveau de l'ouverture où sera placé le matériau à caractériser est une onde plane, à savoir uniforme sur la surface l'ouverture. Cette hypothèse est vérifiée en zone lointaine et permet de confondre les valeurs d'efficacité de blindage en champ électrique, en champ magnétique et, en puissance. Pour cela, une mesure de champ électrique est effectuée à l'aide d'une sonde isotrope. Cette sonde (figure 8) permet de déterminer, pour une fréquence donnée, les trois composantes cartésiennes du champ électrique comme illustré figure 7.

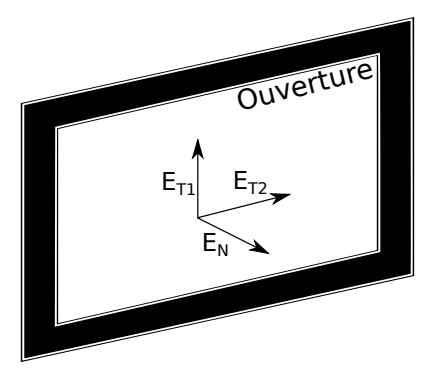

FIGURE 7 - Composantes du champ électrique au niveau de l'ouverture.

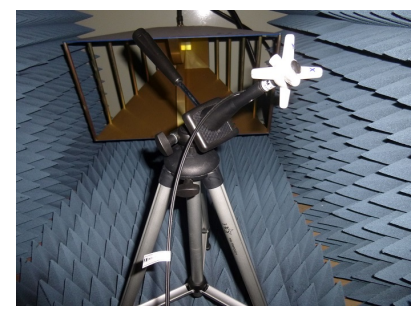

FIGURE 8 - Sonde isotrope.

Les mesures du champ électrique ont été effectuées au centre de l'ouverture pour deux tailles d'ouvertures $(26 \mathrm{~cm}$ et $36 \mathrm{~cm}$ ) et, dans un coin de l'ouverture de $26 \mathrm{~cm}$.
Dans un premier temps, si l'on se concentre sur la vérification de l'hypothèse d'onde plane, nous allons nous intéresser aux valeurs de la norme du champ électrique en deux points de l'ouverture de $36 \mathrm{~cm}$ (au centre et dans un coin) afin de vérifier que l'ouverture plane constitue un front d'onde. Dans ce cas, pour que les seules propriétés de l'antenne d'émission soient prises en compte, des absorbants sont placés sur l'ensemble de l'ouverture.

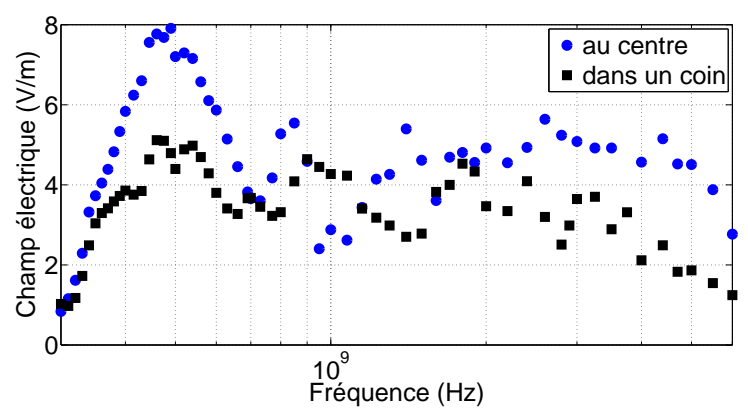

FIGURE 9 - Valeurs du champ électrique en deux points de l'ouverture de $26 \mathrm{~cm}$.

En basses fréquences (jusqu'à $1 \mathrm{GHz}$ ), la longueur d'onde étant très grande (supérieure à $30 \mathrm{~cm}$ ), nous ne vérifions pas la condition de champ lointain. En effet, l'antenne est espacée de l'ouverture d'une distance $d \approx 1 \mathrm{~m}$. En revanche, pour des fréquences plus hautes, la condition de champ lointain est vérifiée. Ainsi, on peut approximer l'onde électromagnétique par une onde plane sur toute la surface de l'ouverture. C'est pourquoi les valeurs de champ électrique dans les deux positions sont assez proches (fig. 9).

De plus, l'onde électromagnétique se propageant dans le vide, elle a la propriété d'être TEM. On présente alors figure 10 les valeurs des composantes du champ électrique tangentielle $E_{T}=\sqrt{E_{T 1}^{2}+E_{T 2}^{2}}$ et normale $E_{N}$ à l'ouverture relativement à sa norme.

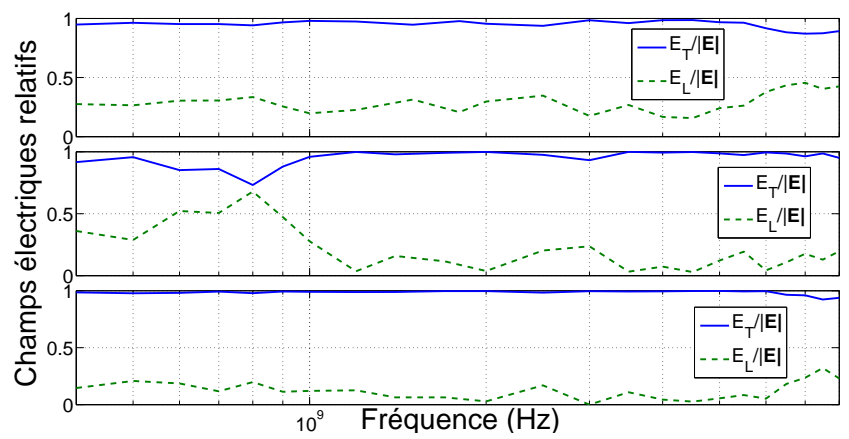

FIGURE 10 - Valeurs des champs tangentiels et normaux dans différents cas. De haut en bas : au centre de l'ouverture de côté $26 \mathrm{~cm}$, dans un coin de l'ouverture de côté $26 \mathrm{~cm}$ et, au centre de l'ouverture de côté $36 \mathrm{~cm}$. 
Nous observons que le champ tangentiel est toujours supérieur au champ normal. De plus, cette propriété caractéristique d'une onde TEM se propageant perpendiculairement à la surface de l'ouverture reste vraie sur tout l'intervalle de fréquence mesuré ( $400 \mathrm{MHz}-8 \mathrm{GHz}$ ). Les mesures correspondant à la bande passante des absorbants, il n'y a pas de réflexions sur les parois de la cellule qui produiraient une composante normale de champ. Cependant, lorsque l'ouverture est trop petite, la composante longitudinale du champ électrique devient non négligeable devant la composante transverse. En effet, elle peut valoir environ 30\% de la norme du champ. L'hypothèse « onde plane » n'est donc plus valable. On remarque également que ce phénomène est renforcé dans les coins de l'ouverture. En effet, l'onde électromagnétique arrivant sur les bords de l'ouverture (tranches, coins) est partiellement diffractée. L'onde ainsi diffractée se propage alors selon une direction non normale à l'ouverture. Sa superposition avec l'onde plane produit alors des composantes de champ électrique dans toutes les directions (notamment une composante normale).

Avant d'effectuer les mesures, l'analyseur de réseau doit être calibré sur la plage de mesure. Cette opération permet de compenser l'impact des câbles et des connections dans les résultats de la mesure.

Une fois le calibrage réalisé, nous pouvons nous intéresser à la dynamique en efficacité de blindage permise par le dispositif. Pour cela, nous devons mesurer le gain à vide du système. Celui-ci dépend de plusieurs paramètres : la position de l'antenne de réception (que l'on peut déplacer contrairement à l'antenne d'émission), la taille de l'ouverture, la présence ou non d'absorbants sur les parois de l'ouverture,... Afin d'obtenir une expérience répétable, tous ces paramètres doivent être fixés et connus.

Les mesures effectuées sont le gain entre antenne d'émission et antenne de réception à vide (appelé $S_{21}^{0}$ ) et, avec blindage (appelé $S_{21}^{b}$ ).

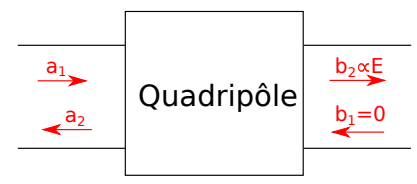

FIGURE 11 - Paramètres mesurés.

La tension délivrée par l'antenne de réception étant proportionnelle aux champs électrique et magnétique (liés par le facteur d'antenne), on a :

$$
\begin{aligned}
E B_{P} & =E B_{E}=E B_{H} \\
& =20 \log \left(\frac{b_{2}^{b}}{b_{2}^{0}}\right) \\
& =20 \log \left(\frac{b_{2}^{b}}{a_{1}^{b}}\right)-20 \log \left(\frac{b_{2}^{0}}{a_{1}^{0}}\right) \\
& =S_{21(d B)}^{0}-S_{21(d B)}^{b},
\end{aligned}
$$

car la puissance générée par l'analyseur de réseau est constante d'où $a_{1}^{b}=a_{1}^{0}$. Il suffit donc de faire la différence entre les mesures de gain à vide et celle en présence du matériau pour déterminer l'efficacité de blindage.

Prenons par exemple, les mesures obtenues à vide et pour l'ouverture de $36 \mathrm{~cm}$ blindée par un grillage (fig. 12).

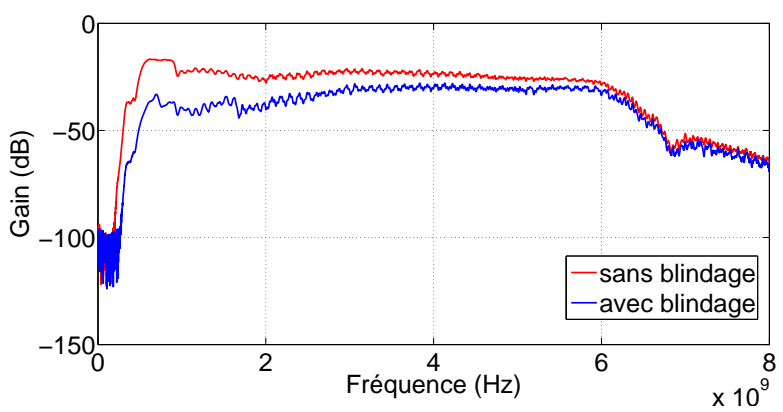

FIGURE 12 - Fonction de transfert du système avec et sans blindage.

A vide, la fonction de transfert du système dépend principalement des caractéristiques des antennes. En deçà de $400 \mathrm{MHz}$, l'antenne émettrice ne rayonne pas suffisamment de puissance pour que la mesure de la puissance reçue soit supérieure au niveau du plancher de bruit. Au delà de $6 \mathrm{GHz}$, les antennes commencent à devenir moins performantes. Ces valeurs correspondent aux données du constructeurs, la bande passante des antennes préconisée allant de $400 \mathrm{MHz}$ à $6 \mathrm{GHz}$.

Comme nous l'avons vu précédemment, le gain minimal que l'on peut mesurer est de $-120 \mathrm{~dB}$. Or, l'atténuation engendrée par le dispositif étant d'environ $20 \mathrm{~dB}$ dans la bande passante, l'efficacité de blindage maximale mesurable est donc de $100 \mathrm{~dB}$.

\subsection{Mesures d'efficacités de blindage}

Les mesures vont être réalisées sur différents matériaux (cf. figure 13) afin de confronter le protocole de mesure à diverses contraintes. 


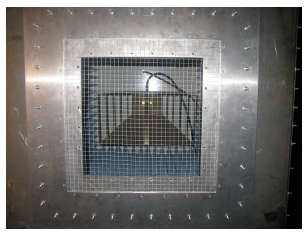

(a) Grillage.

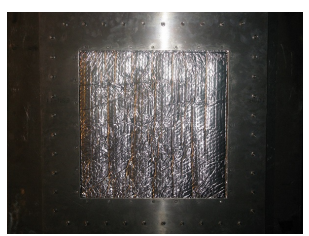

(b) Isolant.

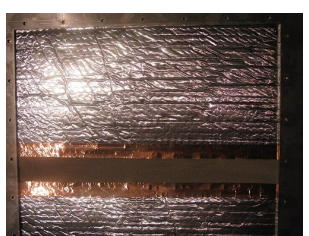

(c) Textile.
FIGURE 13 - Blindages placés sur l'ouverture.

\subsubsection{Efficacité de blindage d'un grillage}

Dans un premier temps, la mesure de l'efficacité de blindage d'un grillage métallique (dont la maille vaut $a=13 \mathrm{~mm}$ et le rayon des fils vaut $r=0,45 \mathrm{~mm}$ ) sera effectuée.

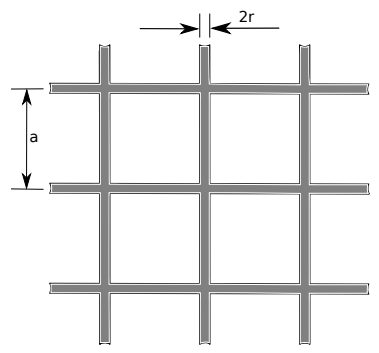

FIGURE 14 - Géométrie du grillage métallique.

Cette mesure peut être comparée à une valeur théorique. On a, pour un grillage illuminé par une onde plane [1] :

$$
E B=20 \log \left(\frac{\sqrt{1+\left(2 \omega L_{s} / \eta_{0}\right)^{2}}}{2 \omega L_{s} / \eta_{0}}\right)
$$

avec $\eta_{0}=\sqrt{\frac{\mu_{0}}{\varepsilon_{0}}}$ l'impédance du vide et, $L_{s}$ l'inductance équivalente du grillage telle que :

$$
L_{s}=-\frac{\mu_{0} a}{2 \pi} \ln \left(1-e^{-2 \pi r / a}\right) .
$$

On présente figure 15 la comparaison entre l'efficacité de blindage mesurée et celle théorique sur une plage de fréquence allant de $300 \mathrm{MHz}$ à $8 \mathrm{GHz}$.

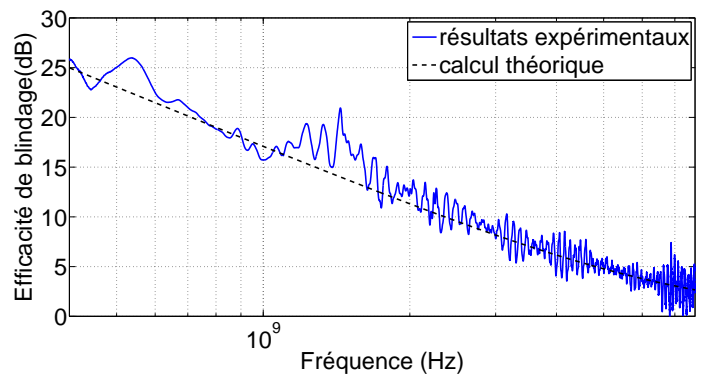

(a) Résultats.

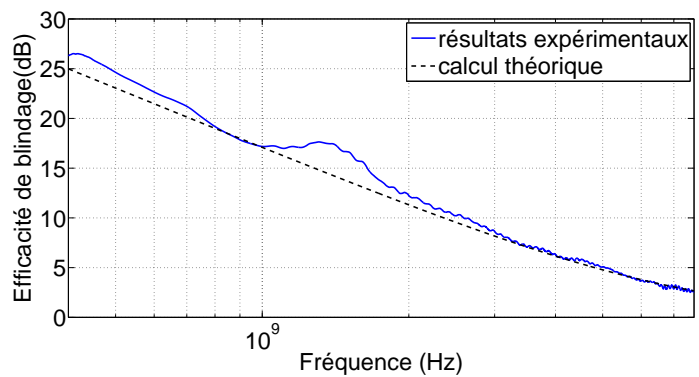

(b) Résultats lissés.

FIGURE 15 - Comparaison entre théorie et résultats expérimentaux de l'efficacité de blindage d'un grillage.

L'efficacité de blindage diminue lorsque la fréquence augmente, cela étant lié au phénomène de pénétration de l'onde par les ouvertures que constituent les mailles du grillage.

Les résultats sont cohérents sur l'ensemble de la plage de mesure. Cependant, quelques différences apparaissent. Aux basses fréquences (inférieures à $700 \mathrm{MHz}$ ), l'efficacité de blindage mesurée est supérieure à celle prévue théoriquement. Cela peut provenir du fait que, à ces fréquences, l'onde n'est pas parfaitement plane et une partie de l'onde illumine le grillage avec une certaine incidence. Or, d'après [1], l'efficacité de blindage d'un grillage illuminé par une onde plane inclinée par rapport au grillage est supérieure à celle où l'onde plane est normale au grillage comme illustré figure 16.

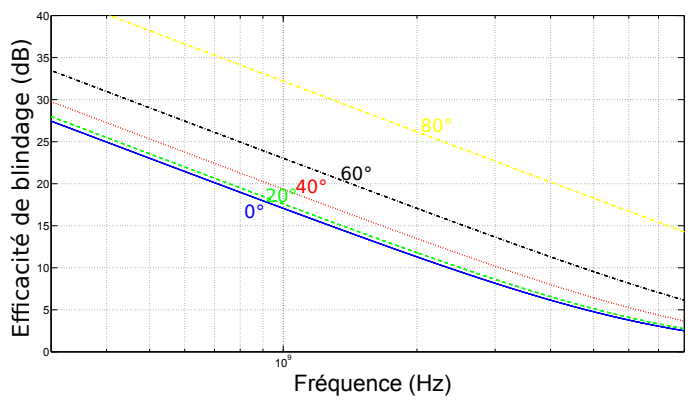

FIGURE 16 - Effet de l'angle d'incidence de l'onde plane sur l'efficacité de blindage du grillage. 
Aux hautes fréquences (supérieures à $1 \mathrm{GHz}$ ), les courbes théoriques et expérimentales sont très proches. Cependant, des oscillations de l'efficacité de blindage allant jusqu'à $5 \mathrm{~dB}$ crête-à-crête apparaissent. Ce phénomène apparaît car le caisson d'émission constitue une cavité résonante, cela signifie que pour les fréquences de résonance de l'enceinte de mesure la majorité de la puissance émise n'est pas absorbée par le caisson. Comme on peut le constater figure 12 , ces oscillations sont plus importantes lorsque le blindage est placé sur les bords de l'ouverture. En effet, les absorbants permettent de limiter ces résonances. Or, dans le cas où le blindage est présent, celui-ci se comporte comme une paroi métallique du caisson d'où l'apparition de résonance (le caisson se comporte comme une cavité résonante).

\subsubsection{Efficacité de blindage d'un matériau métallique}

Dans ce cas, le protocole de mesure est très important. En effet, plusieurs types de fixation de l'échantillon de matériau à tester peuvent donner des résultats de mesure extrêmement variables. Le matériau testé est constitué de deux feuilles métalliques séparées par du papier bulle. Contrairement au grillage, ce blindage ne présente pas d'ouverture. Ainsi, une onde électromagnétique traversant le blindage doit nécessairement traverser les parties métalliques conductrices. Il s'avère donc primordial que le blindage soit en contact parfait avec le caisson. Dans le cas contraire, les résultats des efficacités de blindage mesurées sont faussés. En effet, comme illustré figure 17, une partie de la puissance sera transmise sans traverser le matériau. En raison de légères ouvertures laissées entre le blindage et le caisson, une partie de l'onde incidente est diffractée par ces ouvertures puis transmise vers l'antenne réceptrice [5].

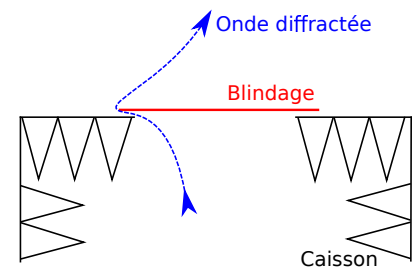

FIGURE 17 - Diffraction d'un partie de l'onde incidente à travers les fuites.

Sur la figure 18(a) ci-dessous, diverses dispositions du blindages ont été testées (isolant fixé à l'aide de vis, fixé entre deux plaques...).

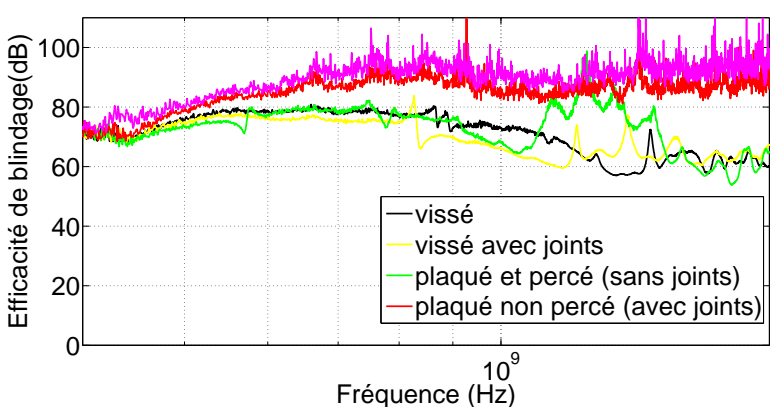

(a) Comparaison des méthodes d'obtention expérimentale de l'efficacité de blindage d'un isolant.

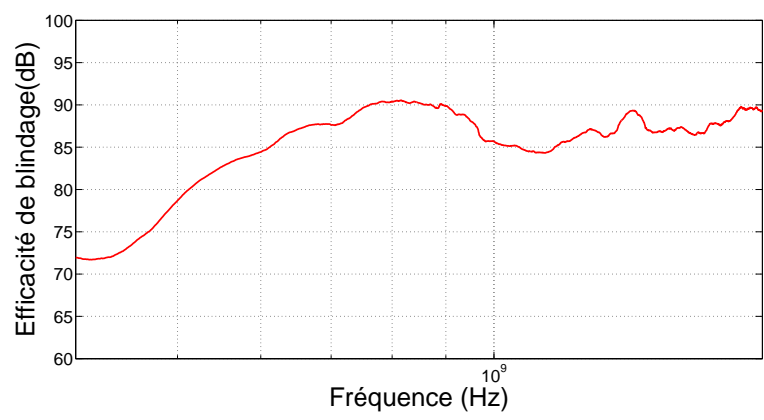

(b) Résultats lissés pour la meilleure méthode.

FIGURE 18 - Efficacité de blindage de l'isolant.

On remarque qu'une méthode nous permet d'obtenir une efficacité de blindage nettement plus importante que les autres. Cette méthode consiste à connecter le blindage au caisson en le fixant entre deux plaques métalliques vissées au caisson (cf. figure 13(b)). Pour assurer une bonne continuité électrique entre les différents éléments et pour limiter les fuites, on ajoute entre les différents éléments des joints conducteurs.

Les méthodes où l'isolant est vissé directement au caisson donnent des résultats qui peuvent présenter des résonances et, une efficacité de blindage plus faible. En effet, de nombreuses ouvertures sont créées à l'emplacement des vis d'où l'apparition de phénomènes de diffraction supplémentaires.

L'efficacité de blindage de ce matériau étant très grande (90 dB au maximum dans la plage de mesure), les mesures du gain blindé $S_{21}^{b}$ sont très faibles et proches du plancher de bruit de l'analyseur de réseau. les résultats sont donc très sensibles aux erreurs de mesure. C'est pourquoi, sur la figure 18(b), la courbe d'efficacité de blindage obtenue par la méthode la plus performante a été lissée.

\subsubsection{Efficacité de blindage d'un matériau textile}

Le matériau textile dont on souhaite connaître l'efficacité de blindage est constitué d'une maille de tissu et de fils conducteurs. Il se présente sous forme de ruban 
de $6,5 \mathrm{~cm}$ de largeur. Les ouvertures disponibles ne permettent de placer que des blindages carrés de $16 \mathrm{~cm}$ de côté au minimum. La méthode alors retenue pour obtenir une mesure de l'efficacité de blindage du textile consiste à créer une fente dans l'isolant précédemment étudié. Le textile est ensuite fixé sur l'isolant à l'aide de ruban adhésif conducteur en cuivre (cf. figure 13(c)). Cette méthode suppose que l'efficacité de blindage du textile soit bien inférieure à celle de l'isolant, pour qu'une grande partie de l'onde transmise le soit au travers du textile.

L'efficacité de blindage est ensuite mesurée pour deux positions de la fente : horizontalement et verticalement. Les résultats sont présentés figure 19.

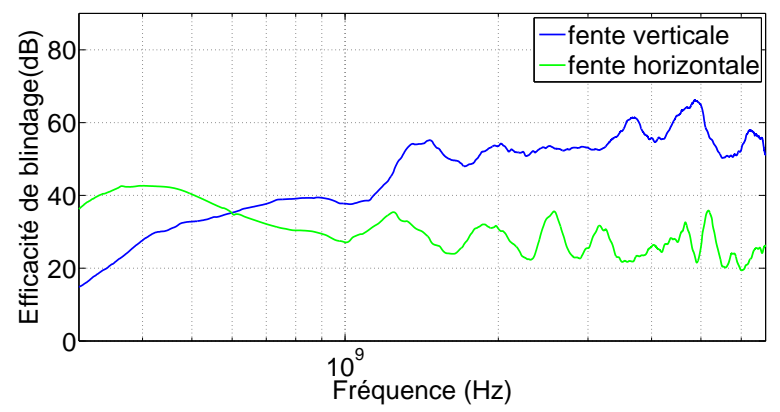

FIGURE 19 - Efficacité de blindage (courbe lissée) mesurée du textile pour les deux positions de la fente.

Le positionnement horizontal ou vertical de la fente ne conduit pas au même résultat. En effet, l'onde générée par l'antenne cornet est polarisée. Ainsi, une fente disposée parallèlement à la polarisation de l'antenne transmettra une puissance bien supérieure à une onde polarisée orthogonalement à celle-ci.

Mais, l'efficacité de blindage étant une mesure relative, les effets dus à la fente doivent être observés de la même manière lorsque la fente est à vide et lorsqu'elle est blindée. L'effet de la polarisation doit donc être compensé. Ce phénomène n'est pas observé car c'est la géométrie du textile qui est responsable de la dispersion des mesures selon la position de la fente. En effet, le tissage du matériau est plus fin dans le sens de la longueur. Cette disposition (cf. figure 20) crée ainsi des fentes dans le sens de la longueur qui auront le même effet que l'ouverture sur laquelle le matériau est placé.

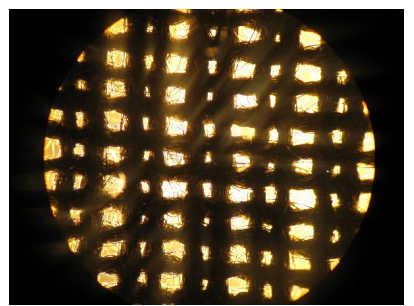

FIgURE 20 - Zoom sur le maillage du textile.

Par conséquent, des matériaux ne possédant pas de symétrie par une rotation de $90^{\circ}$ n'auront pas la même efficacité de blindage mesurée selon leur disposition dans l'ouverture.

Une méthode pour connaître l'efficacité de blindage la plus pénalisante du matériau serait de générer une onde électromagnétique isotrope qui illumine le matériau selon toutes les directions. Ce type d'environnement peut être généré en chambre réverbérante à brassage de modes.

\section{LA CHAMBRE RÉVERBÉRANTE À BRASSAGE DE MODES}

Une deuxième méthode de mesure d'efficacité de blindage va être réalisée au sein d'une chambre réverbérante à brassage de modes (CRBM).

\subsection{Principe}

La CRBM est une enceinte constituée de parois métalliques et munie d'un brasseur de modes, équipement métallique de grandes dimensions (par rapport à la longueur d'onde) en rotation[3]. Une antenne émettrice (voir la figure 21(a)) est placée à l'intérieur de la CRBM, elle est connectée à un analyseur de réseau situé à l'extérieur de la CRBM (voir la figure 22). Celui-ci constitue la source de puissance de l'antenne. Le champ ainsi généré par l'antenne va alors avoir pour propriété d'être statistiquement homogène et isotrope sur une révolution du brasseur et, dans un volume utile situé dans la CRBM. En effet, du fait des réflexions multiples subies par l'onde électromagnétique contre les parois de la CRBM et les pales métalliques du brasseur de mode (figure 21(b)), elle ne possède pas d'orientation privilégiée. La CRBM étant une cavité résonante, les valeurs des fréquences de résonance dépendent de la géométrie de la CRBM. En effet, ce sont les conditions aux limites imposées par les parois métalliques et les pâles du brasseur de modes qui déterminent les valeurs de ces fréquences de résonance. Le brasseur de modes, lors de sa mise en rotation, modifie alors ces conditions aux limites et donc, les valeurs des fréquences de résonance. Cet équipement contribue à l'amélioration du moyen de mesure, lié, à une fréquence donnée, au recouvrement des 
courbes de résonance dues aux pertes dans la structure et à la modification de la cartographie du champ électromagnétique d'une position à l'autre du brasseur.

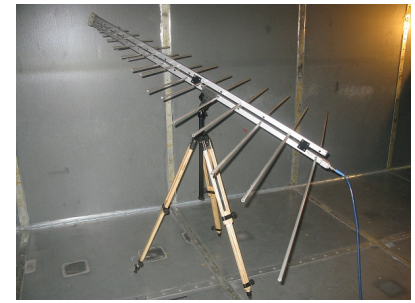

(a) Antenne d'émission.

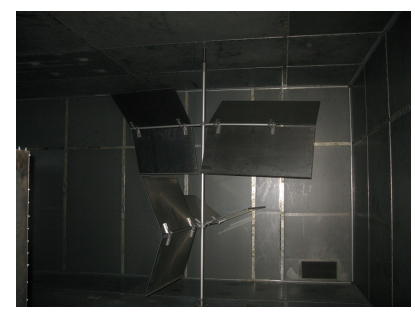

(b) Brasseur de mode.

FIGURE 21 - Éléments de la CRBM.

La figure 22 décrit la configuration dans laquelle est réalisée la manipulation. Le caisson blindé est placé dans la zone utile de la CRBM. Ainsi, l'équipement immergé dans le volume d'essai de la CRBM est soumis à un nombre infini d'ondes électromagnétiques planes de polarisation et d'incidence aléatoires. L'antenne située dans le caisson reçoit alors une partie de l'onde électromagnétique ayant pénétré à l'intérieur du caisson par l'ouverture. En effet, l'onde électromagnétique traversant les parois du caisson est négligeable, celles-ci étant constituées de métal et d'absorbants. Enfin, cette antenne est connectée à l'analyseur de réseau en vue de réaliser des mesures de coefficients $S_{21}$.

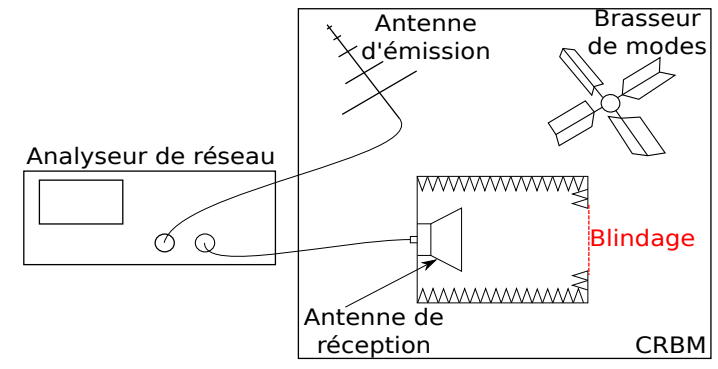

FIGURE 22 - Schéma de principe de la manipulation.

Afin d'exciter toutes les fréquences mesurées (de $400 \mathrm{MHz}$ à $2 \mathrm{GHz}$ ), les mesures de coefficients $S_{21}$ sont effectuées pour 36 positions différentes du brasseur de modes (rotations successives de $10^{\circ}$ ). On évalue alors l'efficacité de blindage par deux formules différentes :

$$
\left\{\begin{array}{l}
E B_{1}=\left\langle S_{21}^{0}\right\rangle-\left\langle S_{21}^{b}\right\rangle \\
E B_{2}=\max S_{21}^{0}-\max S_{21}^{b}
\end{array}\right.
$$

où $\left\langle S_{21}^{0}\right\rangle$ et $\left\langle S_{21}^{b}\right\rangle$ (respectivement $\max S_{21}^{0}$ et $\max S_{21}^{b}$ ) correspondent aux moyennes (respectivement valeurs maximales) des paramètres $S_{21}$ mesurés sur les 36 positions (à vide et blindé).

La première formule doit permettre de limiter les erreurs de mesures dues aux faibles puissances mesurées pour une fréquence donnée selon la position du brasseur de modes. La deuxième permet de retenir la configuration la plus pénalisante pour le blindage, c'est-à-dire celle où le blindage est le moins efficace.

\subsection{Mesures d'efficacités de blindage}

Les mesures d'efficacité de blindage sont effectuées sur le grillage dont on connaît l'efficacité de blindage théorique. Les résultats présentés figure 23 correspondent aux efficacités de blindage mesurées en CRBM dont on a lissé la courbe (moyenne glissante sur $40 \mathrm{MHz}$ ).

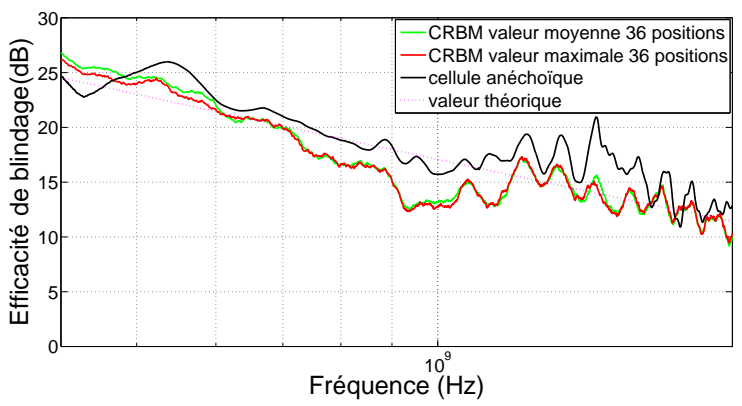

FIGURE 23 - Comparaison entre théorie et résultats expérimentaux de l'efficacité de blindage d'un grillage.

Les résultats sont cohérents avec ceux obtenus à l'aide du caisson anéchoïque et avec les valeurs théoriques de l'efficacité de blindage. L'onde électromagnétique étant constituée d'une superposition d'ondes planes polarisées selon toutes les directions, l'efficacité de blindage mesurée à l'aide de la formule donnant $E B_{1}$ doit correspondre à celle qui est la plus pénalisante selon toutes les incidences. Comme vu précédemment, le cas le plus défavorable correspond à l'onde d'incidence normale au grillage. C'est pourquoi, les résultats montrent que l'efficacité de blindage mesurée reste globalement inférieure à celle mesurée avec la cellule anéchoïque, notamment pour les basses fréquences (inférieures à $1 \mathrm{GHz}$ ) où la cellule anéchoïque ne permet pas d'avoir une onde incidente normale au plan du grillage. 


\section{Conclusion}

Deux méthodes de mesure d'efficacité de blindage ont été étudiées. Ces méthodes peuvent être utilisées pour déterminer l'efficacité de blindage de matériaux plans. La contrainte imposée par le dispositif sur la géométrie des matériaux est de pouvoir disposer d'un échantillon carré permettant de couvrir une ouverture d'au moins $26 \mathrm{~cm}$ de côté.

La comparaison des deux méthodes permet de définir les avantages et inconvénients de chacune, résumés dans le tableau 1.

La cellule anéchoïque permet de mesurer des efficacités de blindage assez importantes (jusqu'à $100 \mathrm{~dB}$ ) et ne nécessite que deux mesures (gain à vide et blindé). Cependant, le blindage n'est caractérisé que pour une onde plane d'incidence normale, qui n'est pas nécessairement le cas le plus défavorable pour le blindage. On peut donc surévaluer l'efficacité de blindage.
La CRBM permet de tester l'échantillon sous toutes les polarisations et, est donc plus pénalisante en terme d'efficacité de blindage. De ce fait, les résultats, basses fréquences notamment, seront plus proches de l'efficacité de blindage réelle que ceux obtenus via la première méthode. En revanche, les mesures sont plus nombreuses (36 mesures dans les deux configurations). D'où des programmes de mesures plus longs et dont la durée doit être prise en compte dans l'organisation des séances de TP. Par ailleurs, la largeur du filtre d'analyse est limitée, ce qui diminue la dynamique de mesure : il est possible de mesurer des valeurs d'efficacité de blindage inférieures à $75 \mathrm{~dB}$ environ.

Finalement, la méthode en cellule anéchoïque est à privilégier pour des mesures rapides et pour mesurer des efficacités de blindage importantes. En revanche, la méthode en CRBM est à privilégier pour effectuer des mesures précises, notamment pour les fréquences situées entre $400 \mathrm{MHz}$ et $1 \mathrm{GHz}$.

\begin{tabular}{|c|c|c|c|}
\hline & $\begin{array}{c}\text { Efficacité de blindage } \\
\text { maximale mesurable }\end{array}$ & Nombre de mesures & Précision \\
\hline Caisson anéchoïque & $100 \mathrm{~dB}$ & 2 & $\begin{array}{c}\text { Surestime l'efficacité } \\
\text { de blindage réelle }\end{array}$ \\
\hline CRBM & $75 \mathrm{~dB}$ & 72 & $\begin{array}{c}\text { Donne l'efficacité de } \\
\text { blindage minimale }\end{array}$ \\
\hline
\end{tabular}

Tableau 1 - Avantages et inconvénients des deux méthodes de mesures.

\section{BIBLIOGRAPHIE}

[1] K.F. CASEY : Electromagnetic shielding behavior of wire-mesh screens. Electromagnetic Compatibility, IEEE Transactions on, 30(3):298-306, Aug 1988.

[2] Pierre Degauque et Bernard Démoulin : Blindages électromagnétiques. Techniques de l'ingénieur, Outils d'analyse en électronique de puissance et métrologie, base documentaire : TIB278DUO.(ref. article : d1320), 2000.

[3] Bernard DÉmoulin et Lamine KonÉ : Mesure de l'atténuation procurée par des blindages. Techniques de l'ingénieur, Outils d'analyse en électronique de puissance et métrologie, base documentaire : TIB278DUO.(ref. article : d1325), 2012.
[4] Keith T. Jacoby, Matthew W. Pieratt, Jennifer I. HALMAn et Keith A. RAMSEY : Predicted and measured emi shielding effectiveness of a metallic mesh coating on a sapphire window over a broad frequency range. SPIE Proceedings, Vol. 7302.(Window and Dome Technologies and Materials X), 2009.

[5] A.C. Marvin, L. Dawson, I.D. Flintoft et J.F. DAWSON : A method for the measurement of shielding effectiveness of planar samples requiring no sample edge preparation or contact. Electromagnetic Compatibility, IEEE Transactions on, 51(2):255-262, May 2009.

[6] Satimo. Dual-ridge horn antennas datasheet, 2014. 